Cahiers québécois de démographie

\title{
Évaluation de la qualité des données par âge et sexe du recensement canadien de 1986
}

EVALUATION OF THE QUALITY OF 1986 CENSUS DATA BY AGE AND SEX AT THE NATIONAL LEVEL EVALUACIÓN DE LA CAUDAD DE LOS DATOS POR EDAD Y SEXO DEL CENSO DE 1986 A NIVEL NACIONAL

\section{Céline Fortier et Ronald Raby}

Volume 18, numéro 2, automne 1989

Sous-dénombrement et estimation de population

URI : https://id.erudit.org/iderudit/010018ar

DOI : https://doi.org/10.7202/010018ar

Aller au sommaire du numéro

Éditeur(s)

Association des démographes du Québec

ISSN

0380-1721 (imprimé)

1705-1495 (numérique)

Découvrir la revue

Citer cet article

Fortier, C. \& Raby, R. (1989). Évaluation de la qualité des données par âge et sexe du recensement canadien de 1986. Cahiers québécois de démographie, 18(2), 285-322. https://doi.org/10.7202/010018ar

\section{Résumé de l'article}

Le recensement est certainement la source d'information la plus complète sur la population canadienne. $\mathrm{H}$ n'est cependant pas exempt d'erreurs, en particulier de l'erreur de complétude. Statistique Canada évalue le niveau de cette dernière erreur, au total et pour certaines caractéristiques, au moyen de la contre-vérification des dossiers. Malheureusement, cette méthode ne permet la mesure que du sous-dénombrement brut. La présente étude a pour but de comparer l'estimation des erreurs de complétude par âge et sexe ainsi obtenues à l'échelle nationale en 1986 à celles qui résultent de la comparaison du recensement à d'autres sources de données : estimations postcensitaires définitives; estimations par composantes (générations nées depuis 1961); et fichiers des récipiendaires d'allocations familiales (0-14 ans) ou de sécurité de la vieillesse (65 ans et plus). L'évolution par âge des niveaux de complétude ainsi calculés est identique d'une source de données à l'autre, même si la valeur des niveaux diffère parfois. La moyenne des valeurs pour chaque âge et chaque sexe a permis, à titre d'exercice, d'estimer l'effectif « réel » de la population canadienne au 1er juin 1986.
Tous droits réservés @ Association des démographes du Québec, 1989
Ce document est protégé par la loi sur le droit d'auteur. L'utilisation des services d'Érudit (y compris la reproduction) est assujettie à sa politique d'utilisation que vous pouvez consulter en ligne.

https://apropos.erudit.org/fr/usagers/politique-dutilisation/ 
Cahiers québécois de démographie Vol. 18, no 2, automne 1989

\title{
Évaluation de la qualité des données par âge et sexe du recensement de 1986 au niveau national
}

\author{
Céline FORTIER et Ronald RABY*
}

\section{INTRODUCTION}

Au 1er juin 1986, date du dernier recensement canadien, la population $^{1} \mathrm{du}$ pays se chiffrait à 25352956 personnes; les femmes étaient légèrement plus nombreuses que les hommes (parts de 50,7\% et 49,3\% respectivement). Les $25-29$ ans étaient les plus nombreux, et ce tant chez les hommes que chez les femmes. Cependant, la structure par âge différait d'un sexe à l'autre, comme le montre l'âge moyen, qui était de 33,3 ans pour les hommes et de 35,4 ans pour les femmes (tableau 1).

Le recensement est de loin la source de données qui foumit, à une date précise, le plus d'informations sur les caractéristiques de la population canadienne. Cependant, comme toute source de données, il est sujet à des erreurs; les plus importantes sont le sous-dénombrement brut (personnes ciblées non dênombrées) et le surdénombrement brut (double compte ou compte de personnes non ciblées) en ce qui concerne la complétude, et les mauvais codages ou les erreurs de réponses ou d'imputation en ce qui a trait à l'exactitude des informations.

Pour évaluer la qualité d'un recensement, il est donc maintenant d'usage d'en calculer soit le degré de complétude

* Statistique Canada, Division de la démographie. Les auteurs, et non Statistique Canada, sont responsables des idées exprimées dans ce texte. Ils aimeraient remercier G. Cartier, M. Michalowski et $\mathbf{M}$. Pouliot, qui ont collaboré à la préparation de cet article.

1 Effectifs recensés au 3 juin 1986 ramenés au ler juin, avec inclusion des effectifs estimés des autochtones vivant sur les réserves partiellement dénombrées. 
TABLEAU 1

Effectifs recensés ${ }^{a}$ et distribution pour 10000 habitants, par âge et sexe, Canada, ler juin 1986

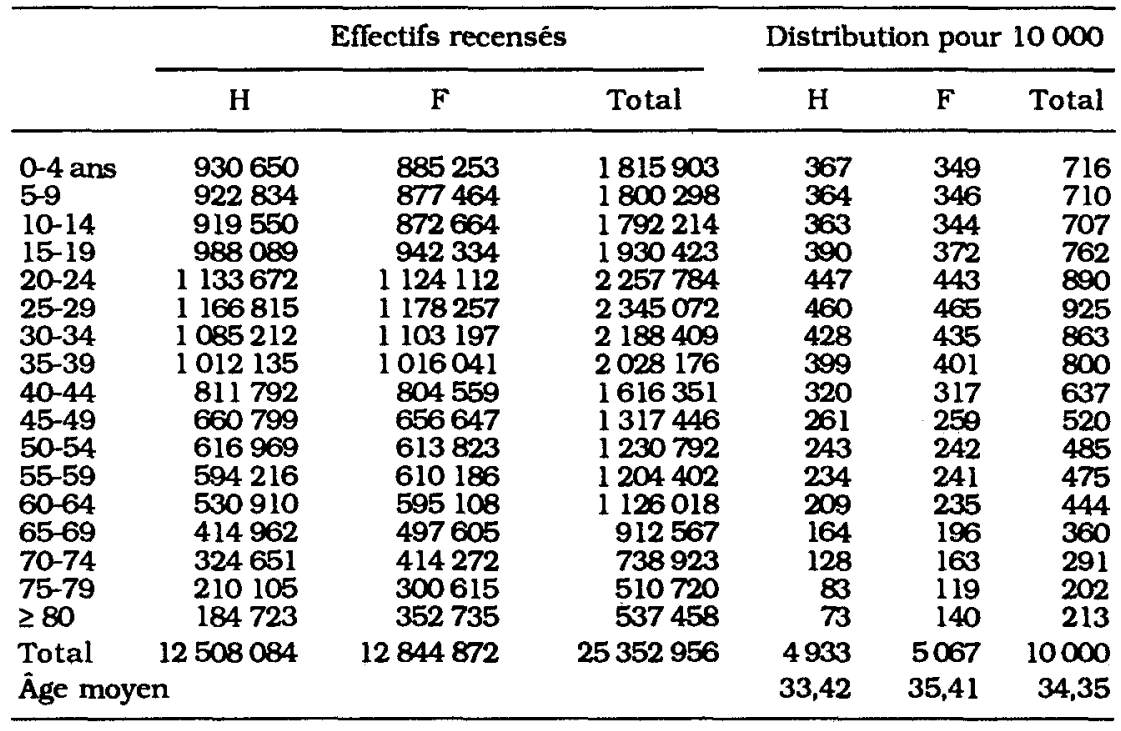

a Effectús recensés au 3 juin 1986, ramenés au ler juin. Inclusion des effectifs estimés des autochtones vivant sur les réserves partiellement dénombrées (estimations intercensitaires calculées à la Division de la démographie de Statistique Canada). Données Inédites.

(qui est simplement la proportion des personnes recensées sur les personnes ciblées), soit l'erreur de complétude (qui est la diffèrence par rapport à $100 \%$ du degré de complétude). En clair, l'erreur de complétude se calcule comme suit :

$$
\frac{\text { Population recensée - Population ciblée }}{\text { Population ciblée }} \times 100 \text {. }
$$

Si l'erreur ainsi obtenue est positive, il y a surdénombrement net du recensement; dans le cas contraire, il y a sousdénombrement net. L'un ou l'autre de ces indices est calculê en premier lieu pour la population totale, mais il peut l'être pour diverses caractéristiques, simples ou combinées, des individus.

Un grand nombre de techniques ont été développées pour êvaluer l'effectif de la population ciblée et donc calculer le degré ou l'erreur de complétude.

1. La tenue d'une enquête postêrieure au recensement, qui, administrée par une équipe indépendante de celle du recensement, refait l'énumération d'un échantillon de la population; 
2. la contre-vérification des dossiers (CVD), qui consiste â rechercher, parmi les personnes recensees, un échantillon indépendant de personnes qui auraient dû l'être. Cet échantillon est composé de répondants et de personnes non énumérées au recensement précédent, d'immigrants arrivés au pays durant la période intercensitaire et d'enfants nés durant cette même période;

3. la méthode dêmographique, qui identifie la population ciblée à la population obtenue par la méthode des composantes en tenant compte des données de migrations, naissances et décès sur une période plus ou moins longue, l'utilisation d'effectifs recensés comme population de départ étant facultative;

4. la comparaison des effectifs recensés à ceux d'autres sources à but statistique, en particulier les registres de population;

5. la comparaison des effectifs recensés à ceux d'autres sources à but non statistique, tels les divers fichiers administratifs d'inscription scolaire, d'assurance-naladie, de permis de conduire, etc.

Statistique Canada mesure la complétude de ses recensements au moyen de la contre-vérification des dossiers. L'exactitude du recensement de 1986 est donc connue selon cette seule approche, et 11 serait intéressant de voir les résultats des quatre autres citées ci-dessus. Malheureusement, Statistique Canada ne tient pas d'enquête postérieure au recensement ${ }^{2}$ et le Canada ne possède pas de registre de population; aussi les approches 1 et 4 sont-elles inutilisables.

Les méthodes 3 et 5, par contre, sont applicables, car les estimations produites par la Division de la démographie de Statistique Canada sont obtenues, au niveau national, par la méthode des composantes; en outre, le Canada dispose de nombreux fichiers administratifs de qualité connue, tels les fichiers nationaux des allocations familiales (pour les 0-14 ans) et de la sécurité de la vieillesse (pour les 65 ans et plus). Dans le cadre d'une analyse provinciale, les fichiers provinciaux, tels ceux de l'assurance-maladie ou des permis de conduire, pourraient aussi être utilisês.

2 Pour la première fois, en 1986, Statistique Canada a tenu une enquête postcensitaire. Son but était, cependant, de mesurer le surdénombrement brut du recensement, à titre uniquement expérimental. L'échantillon étant petit, les résultats sont considérés comme sujets à caution (Statistique Canada, 1988a : 3). 
Cette étude a donc pour but de mesurer le degré de complétude de la population canadienne, par âge et sexe, telle qu'elle a été recensée au ler juin 1986, au moyen de la contrevérification des dossiers, de la méthode démographique et de la comparaison avec deux fichiers administratifs : celui des allocations familiales et celui de la sécurité de la vieillesse. Chaque source de données utilisée sera présentée et leurs différences mises en lumière. Puis les effectifs correspondants seront ajustês afin, d'une part, que leur comparabilitê soit assurée, et. d'autre part, qu'ils se rapprochent autant que possible de l'effectif "réel" de la population. Ils serviront alors à calculer le degré de complëtude du recensement de 1986, par âge et sexe, au niveau national. Enfin, après avoir tenté d'expliquer les écarts entre niveaux de complêtude, nous essaierons d'évaluer, à titre d'exercice, l'effectif "réel" de la population canadienne, par âge et sexe, au ler juin 1986.

\section{SOURCES DE DONNEES UTILISEES}

Si le recensement de la population est une source de données relativement bien connue, il n'en va pas de même des sources tierces que nous désirons utiliser : contre-vérification des dossiers, estimations de la population, fichiers des allocatíons familiales et de la sécurité de la vieillesse de Santé et Bien-être social Canada. À quoi correspondent les effectifs de population qu'elles génèrent ? Quelle est la population ciblée ? Comment est-elle obtenue? Afin d'assurer la comparabilité des effectifs du recensement et de ceux qui sont visés par ces sources, nous nous devons de commencer notre étude par une brève présentation de chacune d'entre elles.

\section{Contre-verification des dossiers}

La contre-vérification des dossiers de 1986 a porté sur un échantillon de 36378 personnes puisé dans quatre sources de données indépendantes du recensement de 1986 : 32200 ont été choisies parmi les personnes dénombrées au recensement de $1981 ; 1776$ parmi les naissances enregistrées à l'état civil entre juin 1981 et fin mai 1986; 1341 parmi les immigrants établis au Canada entre les deux recensements, selon les dossiers d'Emploi et Immigration Canada; et enfin 1061 parmi les personnes ciblées en 1981 mais non recensées, telles qu'elles ont été identifiées par la contre-vérification des dossiers de 1981 (Statistique Canada, 1988a). 
TABLEAU 2

Classification des personnes retenues dans l'échantillon de la contre-vérification des dossiers (CVD) de 1986. par source d'échantillonnage (\%)

\begin{tabular}{lrrrrr}
\hline & Recensées & $\begin{array}{c}\text { Non } \\
\text { recensées }\end{array}$ & Décédées & Émigrées & $\begin{array}{c}\text { Non } \\
\text { dépistées }\end{array}$ \\
\hline Recensement de 1981 & 88,7 & 4,1 & 3,5 & 0,9 & 2,8 \\
Naissances 1981-1986 & 89,3 & 2,0 & 0,6 & 0,9 & 7,2 \\
Immigrants 1981-1986 & 64,9 & 8,6 & 0,7 & 7,2 & 18,6 \\
CVD de 1981 & 71,3 & 12,4 & 2,9 & 3,5 & 9,9 \\
Total & 87,3 & 4,4 & 3,3 & 1,2 & 3,8 \\
\hline
\end{tabular}

Source : Statistique Canada, $1988 \mathrm{a}: 7$.

Quelques jours après la date du recensement, on a entrepris diverses opérations de dépistage afin d'identifier l'adresse de chacune de ces personnes au 3 juin et de déterminer si elle avait été recensée ou non. Cette recherche, cas par cas, a permis de classer chaque individu de l'échantillon dans l'une ou l'autre des catégories suivantes : recensée en 1986; non recensée en 1986; décédée avant le recensement; émigrée avant le recensement; non dépistée. Les résultats figurent au tableau 2.

On a ensuite pondéré ces résultats, d'une part pour tenir compte des individus non retracés, d'autre part pour assurer la cohérence avec les totaux connus de la population. Les poids étaient fonction des caractéristiques, soit la province en 1981, l'âge, le sexe, l'année de naissance, etc. Enfin, on a estimé l'effectif des personnes non recensées, pour diverses variables, en sommant les poids ajustés.

La population ciblée par la contre-vérification des dossiers est identique à celle du recensement. En effet, sont exclus des deux sources les résidents étrangers, qu'ils soient diplomates, membres des forces armées, étudiants, travailleurs temporaires ou visiteurs, et leur famille. Par contre, sont inclus les membres des forces armées canadiennes, les diplomates canadiens et le personnel des navires marchands en poste hors du Canada à la date du recensement (Statistique Canada, 1988b).

De par sa conception, cette méthode ne mesure que le sousdénombrement brut. En 1986, pour la première fois, Statistique Canada a entrepris une étude afin d'estimer le surdénombrement brut. Cette étude exploratoire ne concernait qu'un très falble échantillon d'individus et, par conséquent, ses résultats ont été jugês peu fiables. Cependant, ils indiquent que 
TABLEAU 3

Estimation de l'erreur de complétude au recensement de 1986, selon l'áge et le sexe, au Canada ${ }^{2}$, sur la base de la contrevérification des dossiers (après correction pour tenir compte du surdénombrement brut)

\begin{tabular}{|c|c|c|c|c|c|c|}
\hline & \multicolumn{3}{|c|}{ Erreur de complétude (\%) } & \multicolumn{3}{|c|}{ Écart type (\%) } \\
\hline & $\mathbf{H}$ & $\mathbf{F}$ & Total & $\mathrm{H}$ & $\mathrm{F}$ & Total \\
\hline $0-4$ ans & $-2,10$ & $-2,22$ & $-2,16$ & 0,67 & 0,60 & 0.48 \\
\hline $5-9$ & $-2,51$ & $-1,89$ & $-2,20$ & 0,50 & 0,53 & 0,37 \\
\hline $10-14$ & $-1,34$ & $-2,28$ & $-1,81$ & 0,31 & 0,48 & 0,31 \\
\hline $15-19$ & $-3,95$ & $-3,39$ & $-3,68$ & 0,75 & 0,83 & 0,60 \\
\hline $20-24$ & $-10,13$ & $-6,93$ & $-8,57$ & 0,59 & 0,71 & 0,45 \\
\hline $25-29$ & $-6,70$ & $-3,57$ & $-5,16$ & 0,54 & 0,58 & 0.41 \\
\hline $30-34$ & $-4,16$ & $-3,44$ & $-3,80$ & 0,73 & 0,63 & 0,56 \\
\hline $35-39$ & $-2,88$ & $-0,85$ & $-1,87$ & 0,62 & 0,30 & 0,36 \\
\hline $40-44$ & $-3,64$ & $-1,84$ & $-2,75$ & 0.71 & 0,54 & 0,46 \\
\hline $45-49$ & $-1,98$ & $-1,14$ & $-1,56$ & 0,71 & 0,50 & 0,41 \\
\hline $50-54$ & $-1,80$ & $-1,78$ & $-1,79$ & 0,94 & 0,54 & 0,49 \\
\hline 55-59 & $-2,38$ & $-2,40$ & $-2,39$ & 0,67 & 0,69 & 0,44 \\
\hline $60-64$ & $-1,10$ & $-1,90$ & $-1,52$ & 0,57 & 0,66 & 0,41 \\
\hline$\geq 65$ & $-1,61$ & $-2,00$ & $-1,84$ & 0,52 & 0,44 & 0.33 \\
\hline Total & $-3,70$ & $-2,72$ & $-3,21$ & 0,16 & 0,16 & 0,16 \\
\hline
\end{tabular}

Sources : Statistique Canada, 1988a : 14; tableaux spéciaux, Statistique Canada, Division de la démographie.

a À l'exclusion du Yukon et des Territoires du Nord-Ouest. Ces régions ont été exclues de la contre-vérification des dossiers. car le dépistage des personnes y est difficile et trop coûteux. On notera que la population des réserves indiennes incomplètement dénombrées est considérée comme s non énumérée , aux fins de ce tableau.

le surdénombrement brut est égal à environ $5,4 \%{ }^{3} \mathrm{du}$ taux de sous-dénombrement brut obtenu par la contre-vérification des dossiers (Statistique Canada, 1990). Afin d'obtenir l'erreur de complétude (surdénombrement brut - sous-dénombrement brut) et en l'absence d'autre information sur la valeur exacte du surdénombrement brut, nous avons réduit de $5,4 \%$ les taux de sous-dénombrement brut obtenus par la contre-vérification des dossiers. Ce faisant, nous énonçons implicitement l'hypothèse que le rapport entre sous- et surdénombrement brut ne varie ni par sexe ni par âge, et qu'il est vraiment égal à 5,4\%.

Le tableau 3 présente l'estimation de l'erreur de complétude de la population au recensement de 1986 selon l'âge et le sexe.

345600 personnes auraient été surdénombrées alors que 839256 auraient été omises. 
au Canada (à l'exclusion du Yukon et des Territoires du NordOuest), sur la base de la contre-vérification des dossiers.

L'erreur de complétude est négative partout. Le sousdénombrement brut total, de $3,38 \%$, est le plus élevé qui ait été observé depuis que le Canada évalue la qualité de ses recensements. Celui des hommes (3.91\%) est supérieur à celui des femmes $(2,87 \%)$.

\section{Méthode démographique}

La méthode démographique, qu'utilisent un nombre croissant de pays (dont l'Angleterre-Pays de Galles, les ÉtatsUnis et l'Australie) pour évaluer la qualitê de leur recensement (Redfern, 1981; Choi, Steel et Skinner, 1988; Fay, Passel, Robinson et Conran, 1988), résulte de la comparaison du recensement à des estimations démographiques à la même date, sous l'hypothèse que ces dernières représentent la population cible "réelle". Cette méthode est utilisée à l'interne à Statistique Canada depuis 1961. On se doit toutefois de citer tout particulièrement deux études, soit celle de É. Lapierre (1971) et celle de A. Romaniuc (1988). Ce dernier auteur a fait porter son examen, entre autres, sur les recensements de 1981 et de 1986, mais l'a restreint à une analyse de la population tous âges et sexes réunis pour les provinces et le Canada au total.

Les estimations démographiques utilisées aux fins de la comparaison sont généralement produites au moyen de la méthode des composantes, conformément à l'équation 1. On obtient donc la population de l'année $t+i$ en ajoutant à la population de base les naissances et immigrants de $t, t+i$, et en retirant de la population de base les décès et émigrants en $t, t+i$. La population de base peut découler soit d'un recensement antérieur, soit d'une autre estimation démographique.

$$
\hat{P}_{t+1}=R_{t}+N_{t, t+1}-D_{t, t+1}+I_{t, t+1}-E_{t, t+1}
$$

où $\quad \hat{\mathrm{P}}_{\mathrm{t}+1}=$ estimation de la population au temps $\mathrm{t}+\mathrm{i}$;

$R_{t}=$ effectifs recensés au temps $t$;

$\mathrm{N}_{\mathrm{t} t+1}=$ nombre de naissances survenues entre $t$ et $\mathrm{t}+\mathrm{i}$;

$D_{t, t+1}=$ nombre de décès survenus entre $t$ et $t+i$;

$I_{t, t+1}=$ nombre d'immigrants reçus au Canada entre $t$ et $t+i$;

$\mathrm{E}_{\mathrm{t} t+1}=$ nombre d'émigrants du Canada entre $t$ et $\mathrm{t}+\mathrm{i}$;

$\mathbf{i}=$ nombre d'années sur lequel porte l'estimation. 
Plus le recensement êtudié est éloigné dans le temps, plus les estimations reposent sur les données chronologiques relatives aux naissances, décès et migrations et moins elles sont entachées de l'imprécision inhérente aux données recensées. En fait, si la série de données fiables est disponible sur une période assez longue, 11 est même possible de produire des estimations basées essentiellement sur les composantes de l'accroissement démographique.

Dans cette étude, nous utilisons la méthode démographique à deux reprises. Dans le premier cas, nous comparons les résultats du recensement de 1986 aux estimations postcensitaires définitives produites par Statistique Canada à partir du recensement de 1981. Dans le deuxième, nous retenons comme population repère celle qui est générée depuis 1961 par la méthode des composantes, sans l'appoint d'aucun recensement.

\section{Estimations postcensitaires définitives}

A Statistique Canada, trois séries d'estimations postcensitaires sont produites au moyen de la méthode des composantes pour une même date : provisoires (délai de quatre mois), mises à jour (délal de douze mois) et définitives (délai de vingt mois) (Statistique Canada, 1987). Puisque la série définitive utilise les composantes les plus prêcises, elle servira de base au calcul du degré de complétude du recensement de 1986.

La population cible de ces estimations est la même que celle du recensement. Cependant, les estimations sont entachées des erreurs de complétude du recensement précédent - sur lequel elles sont basées - et donc ne représentent pas cet effectif "rêel". Elles ne le feront que si l'on peut rectifier les effectifs de départ en corrigeant les erreurs de complétude, comme le montre l'équation 2.

$$
P_{t+1}^{\prime}=\left[R_{t}+O_{t}-S_{t}\right]+A_{t, t+1}
$$

où $P_{t+1}^{\prime}=$ population réelle au temps $t+i$;

$R_{t}=$ effectifs recensés au temps $t$;

$\mathrm{O}_{\mathrm{t}}=$ nombre de personnes omises au recensement tenu au temps t;

$S_{t}=$ nombre de personnes surdénombrées au recensement tenu au temps t;

$A_{t, t+1}=$ accroissement de la population survenu depuis le recensement tenu en $t$, jusqu'à $t+i$ (naissances, décès et migrations de l'équation 1);

$i=$ nombre d'années sur lequel porte l'estimation. 
Le nombre de personnes omises au recensement de 1981 est connu, tant par sexe que par groupe d'âge. Par contre, le nombre de personnes surdénombrées ne l'est pas et ne pourra qu'être estimé de façon très grossière, par l'application au nombre de personnes omises en 1981 de la proportion que représente le surdénombrement brut par rapport au sous-dénombrement brut en 1986 (5,4\%). Une fois calculēs, ces effectifs pourront, par addition ou soustraction, selon les cas, servir à rectifier les estimations produites par âge et sexe au ler juin 1986, l'ajustement touchant le groupe d'âge atteint à cette date, soit le groupe de cinq ans plus vieux qu'en 1981. Il est à noter qu'aucune modification n'est apportée aux dècès et migrations, les composantes prises en compte dans le calcul des estimations concernant la population totale, recensée correctement ou non.

Les estimations postcensitaires définitives ainsi ajustèes pour le ler juin 1986 sont présentêes au tableau 4.

\section{TABLEAU 4}

Estimations postcensitaires définitives de la population canadienine basées sur le recensement de 1981, après correction des erreurs de complétude, par âge et sexe, au ler juin 1986

\begin{tabular}{lrrr}
\hline & Hommes & Femmes & Total \\
\hline $0-4$ ans & 954891 & 905814 & 1860705 \\
$5-9$ & 930961 & 882983 & 1813944 \\
$10-14$ & 927972 & 879937 & 1807909 \\
$15-19$ & 1003912 & 954653 & 1958565 \\
$20-24$ & 1229132 & 1183810 & 2412942 \\
$25-29$ & 1259101 & 1249672 & 2508773 \\
$30-34$ & 1121655 & 1125424 & 2247079 \\
$35-39$ & 1046715 & 1039492 & 2086207 \\
$40-44$ & 841947 & 812835 & 1654782 \\
$45-49$ & 687420 & 666221 & 1353641 \\
$50-54$ & 627001 & 619891 & 1246892 \\
$55-59$ & 605536 & 618754 & 1224290 \\
$60-64$ & 538985 & 604711 & 1143696 \\
$65-69$ & 420780 & 499335 & 920115 \\
$70-74$ & 330598 & 420692 & 751290 \\
$75-79$ & 215300 & 307657 & 522957 \\
$\geq 80$ & 189772 & 361502 & 551274 \\
Total & 12931678 & 13133383 & 26065061 \\
\hline
\end{tabular}

Source : Statistique Canada, Division de la démographie, données inédites. 
Estimations démographiques par composantes (population née depuis 1961)

Cette lacune des estimations postcensitaires définitives est éliminée lorsque l'on utilise des estimations démographiques ne faisant intervenir que les composantes de l'accroissement de la population, soit les naissances, les décès et les migrations.

Le Canada dispose d'une série statistique continue des naissances et des décès depuis $1921^{4}$. Puisque l'enregistrement de ces événements à l'état civil est obligatoire. les données finales devraient être de bonne qualitê et couvrir la totalité des personnes concernées. Malheureusement, certaines d'entre elles sont omises par suite de retards dans les inscriptions (formulaires reçus après la date limite du 30 avril).

La qualitê et la disponibilité des données d'immigration sont également très bonnes. Avant leur arrivée au Canada, tous les immigrants reçus doivent remplir le formulaire "Visa d'immigrant et fiche relative au droit d'établissement". Les données statistiques pertinentes sont compilées par Emploi et Immigration Canada.

L'émigration ne faisant l'objet d'aucun enregistrement obligatoire, le nombre d'émigrants doit être estimé de façon indirecte. Différentes méthodes et diverses sources de données ont été utilisées au fil des ans par Statistique Canada (Statistique Canada, 1987). Malgré le raffinement des techniques et la pertinence des sources employées, l'émigration demeure la composante la moins fiable des quatre présentées.

Ces quatre séries de données ont servi à produire, au moyen de la méthode des composantes par cohorte, des estimations démographiques au 1er juin 1986. Les données relatives aux différentes composantes ont été utilisées telles quelles, à l'exception des naissances, que nous avons ajustées pour prendre en compte le sous-enregistrement, parfois élevé. Les estimations ne portent que sur les personnes âgées de 0 à 24 ans, par sexe, puisque les données de migrations pertinentes ne nous étaient facilement disponibles que depuis 1961. Les résultats figurent au tableau 5.

La population générée par ces estimations est composite. D'une part, l'univers couvert par l'êtat civil (naissances et décès) est plus vaste que celui du recensement, puisque sont inclus les

4 Le Guébec et Terre-Neuve se sont joints au système d'enregistrement national d'état civil en 1926 et en 1950 respectivement. 
TABLEAU 5

Estimations démographiques par composantes de la population canadienne née depuis 1961, par âge et sexe, au ler juin 1986

\begin{tabular}{lrrl}
\hline & Hommes & Femmes & Total \\
\hline $0-4$ ans & 958467 & 908464 & 1866931 \\
$5-9$ & 939624 & 893416 & 1833040 \\
$10-14$ & 931164 & 883185 & 1814349 \\
$15-19$ & 999657 & 953005 & 1952662 \\
$20-24$ & 1198288 & 1157338 & 2355626 \\
\hline
\end{tabular}

Source : Statistique Canada, Division de la démographie, données inédites.

événements qui ont touché les étudiants et travailleurs étrangers en séjour temporaire au Canada et les citoyens canadiens et immigrants reçus séjournant plus d'un an à l'étranger. D'autre part, les statistiques d'immigration ne concernent que les immigrants admis à s'êtablir de façon permanente au Canada et excluent donc les citoyens canadiens ou les immigrants reçus qui reviennent au pays après un long séjour à l'étranger, ainsi que les visiteurs, étudiants et travailleurs temporaires étrangers présents au Canada. Quant aux statistiques d'émigration, elles ne visent, en principe, que les citoyens canadiens et les immigrants reçus qui quittent définitivement le Canada. Mais le biais généré n'est pas important.

\section{Fichiers administratifs}

Le Canada ne dispose d'aucun fichier administratif couvrant la population de tous les groupes d'âge. Notre analyse ne pourra donc porter que sur des gênérations spécifiques : les jeunes pour lesquels des allocations familiales (AF) sont versées et les retraités admissibles à la pension de la sécurité de la vieillesse.

\section{Allocations familiales}

En vertu de la loi de 1973 sur les allocations familiales, une redevance est versée mensuellement à tous les enfants de 0 à 17 ans résidant au Canada et "entretenus entièrement ou pour une grande part par un parent " (Loi de 1973 sur les allocations familiales, ch. $44: 7$ ), à condition qu'au début du mois en question au moins un des parents soit résident du Canada, soit comme citoyen canadien, soit comme immigrant reçu, soit enfin comme visiteur ou détenteur d'un permis valide au moins 
un an, assujetti au paiement d'impôts au Canada. De par la loi. les diplomates étrangers sont exclus du programme (Loi des allocations familiales, révision du 15 août 1980 : 6). Sont considérés comme résidents canadiens les personnes qui quittent le pays pour une durée inférieure à un an (vacances, traitement médical, études) et celles qui le quittent indéfiniment mais continuent à payer des impôts au Canada (diplomates, membres des forces armées, personnel de valsseaux canadiens entre autres) (Loi de 1973 sur les allocations familiales, ch. $642: 5$ ).

En 1978, le ministère de la Santé et du Bien-être social du Canada, qui est responsable de l'application de la loi sur les allocations familiales, a créé un ensemble complet de fichiers statistiques sur les 0-17 ans pour qui une allocation est versée. Ces fichiers classent les récipiendaires en plusieurs catégories de types de comptes: réguliers "domestiques" (adresse au Canada); réguliers étrangers; spéciaux agences; spéciaux pour parents nourriciers. Chacune de ces catégories correspond, à peu de chose près, à un segment de la population ciblée par le recensement : leur totalisation devrait donc concerner plus ou moins l'ensemble des individus recherchés. Mais plusieurs catégories de récipiendaires d'AF ne sont pas ciblées par le recensement : les enfants des visiteurs et des étudiants êtrangers séjournant depuis au moins un an au Canada s'ils paient de l'impôt; les enfants des travailleurs temporaires étrangers s'ils ont travaillé au Canada pendant plus d'un an; les enfants des êtudiants canadiens mariés en sêjour à l'étranger; ceux des travailleurs canadiens partis à l'êtranger depuis plus d'un an s'ils paient toujours leurs impôts au Canada; et enfin les enfants de Canadiens en visite de longue durée à l'étranger en raison de vacances ou de traitement médical. Les effectifs des allocations familiales comprennent donc plus d'individus que le recensement et devront être ajustés en conséquence.

La très grande qualité des fichiers d'allocations familiales a été démontrée à plusieurs reprises (Haché, 1984; Michalowski, 1988). Les seules lacunes concernent les jeunes de 16 et 17 ans, dont l'admissibilité peut être remise en cause en cas d'adhésion à la population active. Pour cette raison, nous restreindrons notre comparaison aux seuls 0-14 ans, par groupe d'âge quinquennal. Malheureusement, les données ne sont pas disponibles par sexe.

Les effectifs par âge des enfants pour qui une allocation familiale est versée sont fournis par deux fichiers: le 
fichier F55 (population identifiée par le code postal de résidence) et le fichier M0023 (population par province). Ce dernier est produit quatre fois pour une même date, le fichier original étant mis à jour aux six mois durant deux ans; plus exhaustif, il servira de base à notre comparaison.

Malheureusement, ce fichier n'est pas complet. En effet, suite à la création, en 1984, de trois bureaux régionaux distincts en Ontario (Toronto, Timmins et Chatham), les effectifs totaux de cette province ne sont plus compilês. Ils le sont cependant pour le fichier F55. On a donc estimé les effectifs par âge des enfants ontariens pour qui une allocation a êté versée en juin 1986 en majorant les chiffres de F55 de la proportion d'enfants ajoutés pour les neuf autres provinces et les deux territoires, entre $\mathrm{F} 55$ et $\mathrm{MOO23}^{5}$. Les enfants pour qui une allocation familiale a été versée en juin 1986 au Canada, par âge, figurent au tableau 6.

TABLEAU 6

Nombre d'enfants pour quit une allocation familiale a été versée, par groupe quinquennal d'âge, juin 1986, Canada

\begin{tabular}{ll}
\hline $0-4$ ans & 1855348 enfants \\
$5-9$ & 1822065 \\
$10-14$ & 1807190 \\
\hline
\end{tabular}

Sources : Fichiers F55 et M0023 (version 4) des allocations familiales, Santé et Bien-être social Canada, et Statístique Canada. Division de la démographie, données Inédites.

Comme nous l'avons expliqué précédemment, ces effectifs ne sont pas directement comparables aux effectifs recensés. Ils le seront si on en déduit les enfants des visiteurs et étudiants êtrangers séjournant depuis au moins un an au Canada et y payant de l'impôt; ceux des travailleurs temporaires étrangers ayant travaillé au moins un an au Canada; ceux des étudiants canadiens séjournant à l'étranger; ceux des travailleurs canadiens partis à l'étranger depuis plus d'un an mais continuant à payer leurs impôts au Canada; et enfin ceux des

5 Pour les âges 1 à 14 inclusivement, l'écart entre les provinces était faible et l'utilisation de l'écart total des deux territoires et neuf provinces autres que l'Ontario se justifie. Pour l'àge 0 , la situation étalt différente, les écarts étant semblables à l'intêrieur des provinces Maritimes et des autres mais différents entre eux. La proportion ajoutée pour l'Ontario a donc été la moyenne de celles des provinces suivantes: Québec, Manitoba, Saskatchewan. Alberta et Colombie-Britannique. 
Canadiens en visite de longue durée à l'étranger pour cause de vacances ou de traitement médical et qui n'ont pas étê recensés.

Le fichier d'enregistrement des visiteurs d'Emploi et Immigration Canada permet d'estimer le nombre d'enfants de 0 à 14 ans résidant au Canada qui ont droit aux allocations familiales mais ne font pas partie de la population ciblée par le recensement. Les résultats préliminaires de l'analyse de ce fichier à Statistique Canada ${ }^{6}$ indiquent que, le ler juin 1986, 4346 enfants de 0 à 14 ans étaient détenteurs d'un permis de visiteur, d'étudiant ou de ministre, parmi un total de 93935 personnes de tous âges détentrices d'un permis de séjour temporaire et présentes au Canada depuis au moins un an. Parmi ces 93935 détenteurs d'un permis de séjour temporaire à long terme, 55858 adultes ont un permis de travail et font donc partie des catégories ciblées par les allocations familiales. Sous l'hypothèse que la fécondité est similaire entre groupes d'étrangers, l'application, aux effectifs d'enfants détenteurs d'un permis temporaire, de la proportion d'adultes ciblés par les allocations familiales dans l'ensemble des adultes dêtenteurs d'un permis temporaire (89 589) nous donne une estimation de 2710 enfants de 0 à 14 ans non recensés pour qui une allocation familiale est versée. Il reste à ajouter les enfants des étudiants étrangers qui reçoivent une bourse du Canada (et qui, eux aussi, doivent payer leurs impôts au Canada); on estime cet effectif en appliquant aux boursiers de l'Agence canadienne de développement international prêsents depuis au moins un an au Canada la proportion enfants/adultes des détenteurs de permis canadiens selon le fichier d'Emploi et Immigration Canada. Au nombre de 61, ces enfants portent à 2771 le total des récipiendaires d'allocations familiales résidant au Canada mais non ciblés par le recensement de 1986.

Les enfants des étudiants, travailleurs et visiteurs canadiens séjournant à l'étranger sont plus difficiles à estimer. D'une part, lls sont additionnés, dans les fichiers des allocations familiales, aux enfants de diplomates ou de militaires, qui sont eux ciblés par le recensement; d'autre part, ils ne font l'objet d'aucun enregistrement exhaustif particulier. Donc. pour éviter les effets d'une mauvaise estimation, nous croyons préférable de limiter notre comparaison aux enfants pour qui une allocation familiale est versée et aux effectifs recensés au

6 Ce projet est dirigé par Madame Margaret Michalowski. 


\section{TABLEAU 7}

Nombre d'enfants ciblés par le recensement pour qui une allocation familiale a été versée au Canada, et nombre d'enfants recensés au Canada, par groupe quinquennal d'âge, juin 1986

\begin{tabular}{lcc}
\hline & $\begin{array}{c}\text { Enfants ciblés par le recensement } \\
\text { pour qui une AF a été versée }\end{array}$ & $\begin{array}{c}\text { Enfants } \\
\text { recensés }\end{array}$ \\
\hline $0-4$ ans & 1851372 & 1813178 \\
$5-9$ & 1818769 & 1798163 \\
$10-14$ & 1804278 & 1790409 \\
\hline
\end{tabular}

a Sources : tableau 6; ajustements de la Division de la démographie de Statistique Canada.

b Sources : Recensement du Canada, 1986, tableaux spéciaux; estimations intercensitaires calculées à la Division de la démographie de Statistique Canada. Données inédites.

Canada seulement. Les deux sources de données doivent donc être ajustées.

Il y aurait au Canada 2771 enfants âgés de 0 à 14 ans pour qui une allocation familiale aurait été versée en juin 1986 , mais qui n'auraient pas été recensés. Cet effectif, déduit des 5477190 enfants rêcipiendaires ce mois-là au Canada, laisse 5474419 enfants récipiendaires visés par le recensement. Si on suppose que la répartition par âge des 2771 enfants omis est la même que celle du total des enfants pour lesquels une allocation a été versée, on obtient la répartition par groupe d'âge des 5474419 enfants récipiendaires d'AF ciblés par le recensement du Canada en juin 1986. Les résultats figurent au tableau 7. Les effectifs des enfants recensés en 1986 sur le territoire du Canada sont également présentés dans ce tableau.

Sécurité de la vieillesse

À l'autre extrèmité de l'êchelle des âges, les retraités aussi ont droit à des prestations de Santé et Bien-être social Canada. Elles sont de trois types : le Régime de pensions du Canada ou le Régime des rentes du Québec ${ }^{7}$, liés à la participation antérieure au marché du travail; le Programme de la sécurité de la vieillesse (PSV), universel et fonction de l'âge et du statut de résidence; et enfin, le Supplément de revenu garanti.

Étant donné sa couverture universelle, le PSV devrait nous permettre de mieux connaître l'effectif des 65 ans et plus au Canada en juin 1986. En vertu de la loi sur la sécurité de la

7 Le Régime de pensions du Canada est appliqué partout au Canada, sauf au Québec; cette province possède son propre régime, la Régie des rentes du Québec. 
vieillesse (1985, ch. 0-9), des prestations mensuelles sont versées, à sa demande, à tout individu âgé de 65 ans et plus, qu'il soit citoyen canadien ou rêsident légal du Canada, à condition qu'il ait résidé, à la date d'agrément de la demande, au moins dix ans au Canada depuis l'âge de dix-huit ans. Si la durée de résidence est inférieure à vingt ans, le demandeur doit également avoir résidé au Canada le jour précédant la date d'agrément de la demande. Le retraité qui émigre ou s'absente temporairement du pays touche tout de même sa pension, pendant six mois s'il a vécu au total entre dix et vingt ans au Canada après ses 18 ans, à vie s'il a vécu au total entre dix et vingt ans au Canada après ses 18 ans (Lois révisées du Canada de 1985, ch. 0-9, par. 3.2). Quant à l'immigrant retraité arrivé au pays depuis molns de dix ans, ll n'a droit à la sécurité de la vieillesse que si son pays d'origine a signé un accord international de sécurité sociale avec le Canadas (Enregistrement DORS/81-285 du 3 avril 1981, ch. 21.5.1 et 21.5.2).

Néanmoins, certaines de ces personnes admissibles ne recevront pas, un mois donné, leur prestation de la sécurité de la vieillesse car :

- elles sont présumées décédées le premier jour du mois (Lois révisées du Canada de 1985, par. 8.3.);

- la demande qu'elles ont présentée n'a pas été agréée le mois précédent ou avant (ibid., par. 8.1);

- elles font l'objet d'une suspension;

- elles manquent aux dispositions de la loi ou de ses règlements (ibid., par. 9.5);

- elles font l'objet d'une enquête quant à leur admissibilité à la sécurité de la vieillesse.

Leur admissibilité établie, les allocations auxquelles ces personnes avalent droit leur seront cependant versées a posteriori. À l'opposé, certains individus recevront des prestations non justifiées, trop-perçu qu'ils devront rembourser par la suite.

Autre détail technique : le premier mois pour lequel est versée une redevance n'est pas celui de l'anniversaire des 65 ans mais le suivant; de même, le dernier mois pour lequel est versée cette redevance est celui du décès.

8 De tels accords existent entre le Canada et l'Autriche, la Barbade, la Belgique, le Danemark, l'Espagne, les États-Unis, la Finlande, la France, la Grèce, l'Italie, la Jamaique, le Luxembourg, la Norvège, les Pays-Bas, le Portugal, la République fédérale allemande, Sainte-Lucie et la Suède. 
Les chèques de sécurité de la vieillesse sont émis, à quelques exceptions près, par les bureaux régionaux responsables. Cependant, "les bénéficiaires de la sécurité de la vieillesse qui se sont qualifiés pour une pension de retraite du Régime de pensions du Canada avant 1970 et qui ne reçoivent pas de supplément de revenu garanti" 9 reçoivent leur paiement conjointement avec celui du Régime de pensions du Canada.

La Direction générale des programmes de la sécurité du revenu de Santé et Bien-être social Canada tient un fichier administratif de toutes ses opérations : le fichier maitre des bênéficiaires. Ce fichier, contrairement aux fichiers relatifs aux allocations familiales, n'est pas réactualisé après coup de façon à inclure les versements a posteriori pour un mois donné. Il ne concerne donc que les versements effectués durant le mois concerné et exclut les cas de suspension, d'annulation, en attente, etc. Il exclut également les paiements d'allocations de vieillesse effectués par le Régime de pensions du Canada. Ce fichier administratif classe les bénéficiaires par âge, sexe, type de compte et province d'imputation du chèque.

Ce fichier sert de base à une série de tableaux statistiques publiés mensuellement dans une brochure émise par Santé et Bien-être social Canada ${ }^{10}$. Les renseignements sont classés en comptes "domestiques" (le lieu de rêsidence du récipiendaire est le Canada) et comptes à l'étranger (le lieu de résidence du récipiendaire est un pays autre que le Canada). Malheureusement, les tableaux publiés ne donnent aucune information sur la répartition par âge et sexe des bénéficiaires, nécessaire à notre analyse. Nous avons cependant pu obtenir de Santé et Bien-être social Canada des tableaux inédits présentant la structure par âge et sexe des bénéficiaires par type de comptes, à l'exclusion des accords de réciprocité et des paiements faits par le Régime de pensions du Canada.

Ces données devront être ajustées. En effet, la population de 65 ans et plus ciblée par le recensement diffère quelque peu de celle qui a droit à la sécurité de la vieillesse et, dans ce sens, les deux séries ne sont pas directement comparables. Reçoivent des prestations de sécurité de la vieillesse mais ne sont pas recensés les émigrants canadiens de 65 ans et plus ayant vêcu

9 Traduction. Tableaux de documentation OAS/GIS/SPA.

10 La brochure s'intitule Statistiques mensuelles, programmes de la sécurité du revenu Régimes de pensions du Canada, allocations famillales, sécurité de la vieillesse. 
au moins vingt ans au Canada depuis leurs 18 ans et ceux qui ont vêcu entre dix et vingt ans au Canada depuis ce même âge s'ils ont quitté le Canada il y a moins de sept mois. Seuls sont recensés, parmi eux, les membres des forces armées canadiennes, les diplomates canadiens et le personnel des navires marchands du Canada. Ne sont pas recensés non plus les récipiendaires d'allocations de sécurité de la vieillesse qui ne sont ni citoyens canadiens ni immigrants reçus mais détiennent un permis de sêjour à long terme au Canada.

Par contre, sont recensés sans recevoir la sécurité de la vieillesse les citoyens canadiens et les immigrants reçus âgés d'au moins 65 ans qui n'ont pas résidé au moins dix ans au Canada après leurs 18 ans, qu'ils soient présents au Canada lors du recensement ou absents car membres des forces armées canadiennes, du personnel diplomatique canadien à l'êtranger ou du personnel de navires marchands canadiens. Les années de rêsidence au Canada, au sens de la sécurité de la vieillesse, comprennent non seulement les années passées au Canada mais aussi les années vécues dans un autre pays avec lequel le Canada a signé un acord de réciprocitê de la sécurité sociale.

Les populations ciblées ne seront donc comparables que si nous utilisons, d'une part, les récipiendaires domestiques de la sécurité de la vieillesse, à l'exception des personnes admissibles en vertu d'accords internationaux et des résidents légaux du Canada qui ne sont ni citoyens canadiens ni immigrants reçus; et, d'autre part, les effectifs recensés, moins les personnes de 65 ans et plus recensées à l'étranger et les immigrants reçus les dix années précédentes et âgés de 65 ans et plus le 3 juin 1986. Pour être précis, 11 faudrait pouvoir exclure également du recensement toutes les personnes retraitées qui ont immigré au Canada avant juin 1976 mais dont les sorties subséquentes du territoire canadien portent la durée totale de résidence au Canada à moins de dix ans. Il est impossible de cerner ces personnes, malheureusement. Cependant, leur effectif est vraisemblablement très faible et devrait être compensé par celui des gens qui ont résidé temporairement au Canada avant de s'y installer comme immigrants reçus après mai 1976 (que nous avons exclus).

Les tableaux relatifs aux récipiendaires par âge et sexe de la sécurité de la vieillesse en juin 1986 (fournis par Santé et Bienêtre social Canada) contiennent l'information recherchée, à l'exclusion toutefois des paiements effectués par le Régime de pensions du Canada. Ces comptes, dont le nombre est cepen- 
TABLEAU 8

Récipiendaires de la sécurité de la vieillesse en juin 1986, par âge et sexe, comptes domestiques, à l'exclusion des personnes admissibles en vertu d'accords internationaux, Canada

\begin{tabular}{lccc}
\hline & Hommes & Femmes & Total \\
\hline $65-69$ ans & 401488 & 478977 & 880465 \\
$70-74$ & 317617 & 401811 & 719428 \\
$75-79$ & 206775 & 293353 & 500128 \\
$\geq 80$ & 179939 & 353990 & 533929 \\
\hline
\end{tabular}

Sources : Santé et Bien-être social Canada, Statistiques mensuelles, programmes de la sécurité du revenu. Régimes de pensions du Canada, allocations familiales, sécurité de la vieillesse, juin 1986, tableau 4; tableau 101, produit à partir du fichier maître des récipiendaires de la sécurité de la vieillesse. Santé et Bien-ètre social Carıada, données inédites. Calculs faits à la Division de la démographie de Statistique Canada.

dant connu (11 187), ne concernent que des récipiendaires âgés de plus de 80 ans (au 1er janvier 1970, ils avaient au moins 65 ans) et cet effectif sera donc ajouté aux effectifs correspondants de récipiendaires de la sécurité de la vieillesse, au prorata des récipiendaires domestiques par sexe (tableau 8).

Pour ajuster les chiffres du recensement, nous avons soustrait des effectifs présentés au tableau 1 : les personnes de 65 ans et plus membres de ménages étrangers; l'ensemble des immigrants reçus âgês de 65 ans et plus arrivés au Canada de 1977 à 1986; et $56 \%$ de ceux qui sont arrivés en 1976 (proportion des immigrants reçus entre juin et décembre dans l'ensemble des immigrants de l'année, selon le rapport annuel d'Emploi et Immigration Canada) d'après des compilations spéciales du recensement de 1986 (tableau 9).

\section{TABLEAU 9}

Effectifs recensés au Canada en juin 1986, à l'exclusion des personnes ayant migré après mai 1976, personnes de

65 ans et plus, par groupe d'âge et sexe, Canada

\begin{tabular}{llll}
\hline & Hommes & Femmes & Total \\
\hline $65-69$ ans & 406184 & 485317 & 891501 \\
$70-74$ & 317858 & 405210 & 723068 \\
$75-79$ & 206606 & 295541 & 502147 \\
$\geq 80$ & 182445 & 348544 & 530989 \\
\hline
\end{tabular}

Sources : Recensement du Canada de 1986, tableaux spéciaux; estimations intercensitaires calculées à la Division de la démographie de Statistique Canada. Calculs effectués à la Division de la démographie de Statistique Canada. Données inédites. 


\section{ANALYSE COMPARÉE DES RESULTATS}

Les effectifs des récipiendaires de la sécurité de la vieillesse ainsi ajustés pourront maintenant être comparés à ceux, également corrigés, des mêmes personnes ciblées recensées en 1986, par le calcul de l'erreur de complétude pour chaque sexe et chaque groupe d'âge. C'est aussi le cas des nombres obtenus des autres sources de données présentées : les estimations postcensitaires définitives et les estimations par composantes pour les générations nées depuis 1961; les allocations familiales. Les différentes séries d'erreurs de complétude ainsi obtenues de même que celle qui résulte de la contre-vérification des dossiers de 1986 (ajustées pour le surdênombrement brut) pourront alors être comparées entre elles. Les données figurent au tableau 10 et sont illustrées aux graphiques 1,2 et 3 .

Les erreurs de complétude n'existent pour l'ensemble des cinq sources que pour les deux sexes reunis, les données d'allocations familiales n'étant pas disponibles par sexe. Nous commencerons donc notre analyse comparative par l'examen des courbes deux sexes réunis (graphique 1).

Deux caractéristiques des courbes frappent au premier coup d'oeil. D'abord, on peut voir qu'à l'exception des groupes d'âge 65-69, 70-74 et 75-79 ans pour la sécuritê de la vieillesse, les erreurs de complétude sont négatives partout, ce qui veut dire que le recensement souffre d'un sous-dénombrement net. Le niveau de ce sous-dênombrement varie cependant d'un groupe d'âge à l'autre mais, et c'est là le deuxième aspect frappant de ces courbes, il est indéniable que l'évolution des erreurs par âge est similaire pour l'ensemble des sources de données. En fait, si l'on tient compte de l'intervalle de confiance entourant chaque donnée, il appert même que les cinq approches se juxtaposent.

L'évolution du niveau des erreurs de complétude par âge serait donc la sutvante : de $-2 \%$ à - $3 \%$ pour le groupe d'âge 0-4 ans, le niveau diminuerait dans les deux groupes d'âge suivants, puis augmenterait rapidement, jusqu'à atteindre son point culminant chez les 20-24 ans, suivis cependant de près par les 25-29 ans. Il diminuerait par la suite à bon rythme chez les 30-34 et les 35-39 ans, pour se stabiliser alors, pour les groupes d'âge suivants, jusque chez les 65-69 ans; là, il recommencerait à croître, mais légèrement.

Cette évolution similaire des erreurs de complétude par âge, de même que des niveaux pour un même groupe d'âge (relativement égaux), masque néanmoins certains écarts importants 


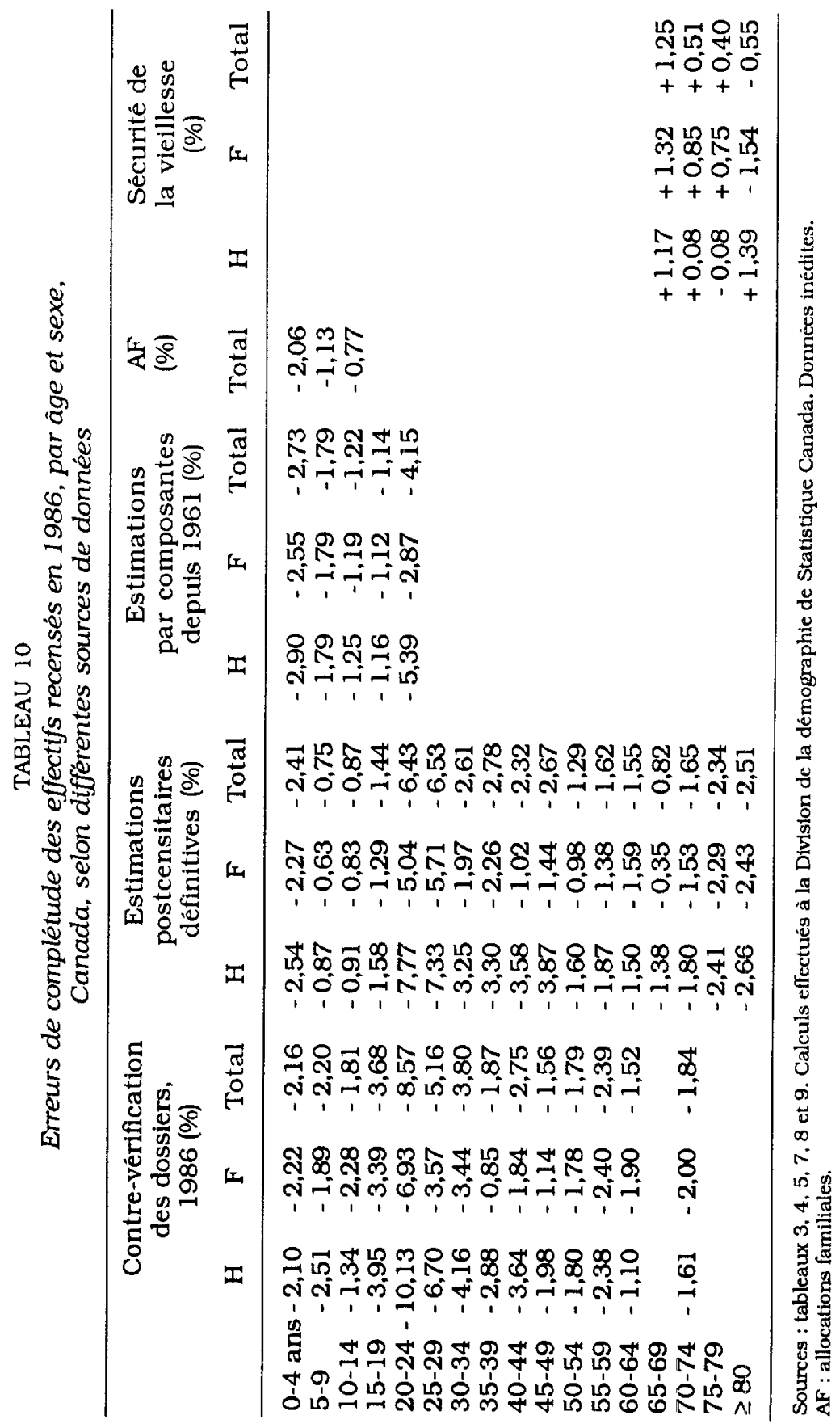


GRAPHIQUE 1 - Erreurs de complétude du recensement de 1986, par àge, différentes sources de données, sexes réunis, Canada

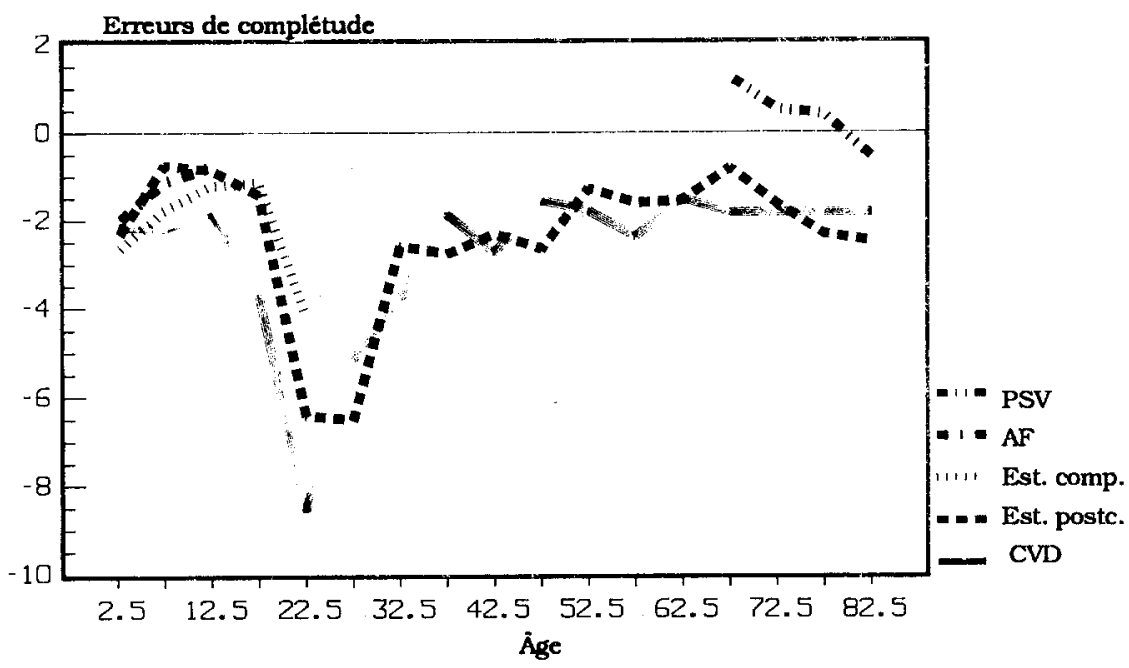

GRAPHIQUE 2 - Erreurs de complétude du recensement de 1986, par âge, différentes sources de données, hommes, Canada

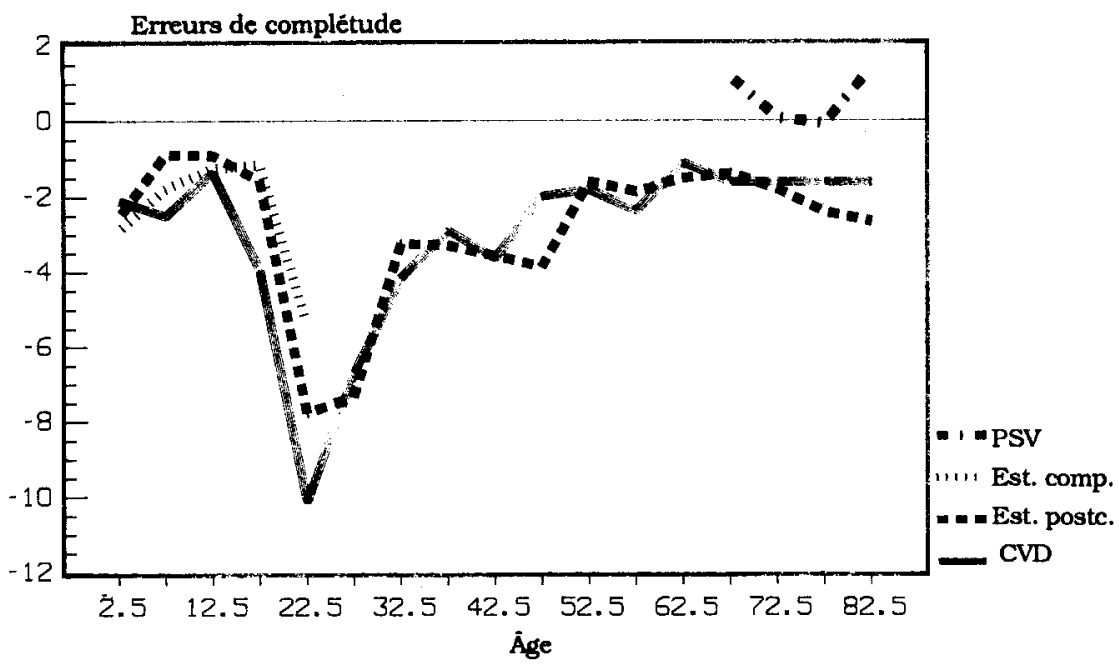


GRAPHIQUE 3 - Erreurs de complétude du recensement de 1986, par âge, différentes sources de données, femmes, Canada

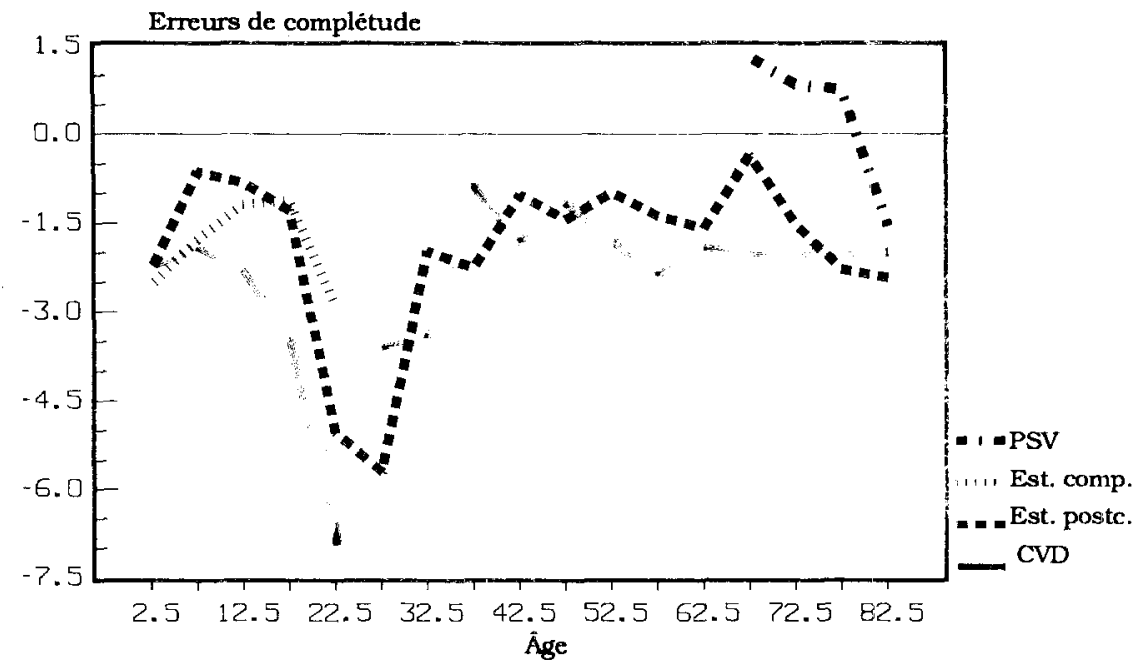

qui pourraient rendre difficile la détermination de la valeur exacte "réelle" du sous-dénombrement net pour chaque groupe d'âge. Le maximum atteint, observé partout à 20-24 ans sauf pour la courbe des estimations postcensitaires définitives (2529 ans), oscille entre 4,15 (estimations par composantes pour la population née depuis 1961) et 8,57 (contre-vérification des dossiers), en passant par 6,53 (estimations postcensitaires définitives). Le niveau va, entre les extrêmes, du simple au double, ce qui n'est pas négligeable. Autre démarcation importante : la courbe de la sécurité de la vieillesse. Pour les quatre groupes d'âge pour lesquels l'erreur a été calculée, elle s'éloigne passablement de celle qui est observée pour les autres sources (de deux points de pourcentage environ), même si son évolution est similaire. De plus, comme nous l'avons écrit précédemment, les trois premiers groupes d'âge présentent une erreur positive, c'est-à-dire un surdénombrement net du recensement (farble cependant). Nous tenterons d'expliquer ces différences dans la prochaine section. Mais auparavant, il importe de faire le point sur l'évolution des erreurs de complétude, non seulement par âge mais également par sexe (graphiques 2 et 3).

Lorsque notre regard embrasse d'un seul coup les trois graphiques, il saute aux yeux que l'évolution de l'erreur de complétude par âge est identique de l'un à l'autre: mêmes 
hausses, mêmes baisses, même maximums aux mêmes âges. Mais les graphiques ne sont pas exempts de dissemblances. Nous soulignerons les trois principales.

Premièrement, si l'évolution est similaire, les niveaux des erreurs pour chaque sexe s'éloignent à certains âges, et très fortement. Ils sont, de 20 à 44 ans, beaucoup plus faibles chez les femmes que chez les hommes, puisqu'ils atteignent respectivement, pour les 20-24, les 25-29, les 30-34, les 35-39 et les $40-44$ ans, environ 5 et 8, 4,5 et 7, 3 et $4,1,5$ et 3 et 1,5 et 3,5.

Ensuite, il convient de souligner que les courbes sont beaucoup plus rapprochées dans le cas des hommes que dans celui des femmes, si l'on excepte les groupes d'âge 15-19, 20-24 et 65 et plus. En fait, la moyenne des écarts entre valeurs extrêmes est de 0,7 point de pourcentage pour les hommes et de 1,0 pour les femmes. Mais cet écart est de faible niveau dans les deux cas.

Enfin, il faut noter que si, tant chez les hommes que chez les femmes, les erreurs de complêtude basées sur les données relatives à la sécurité de la vieillesse se démarquent nettement de celles des autres sources, leur allure par âge est différente chez les hommes, puisqu'elle subit une hausse marquée chez les 80 ans et plus, passant de $-0,08 \%$ pour les $75-79$ ans à $+1,39 \%$.

La similitude de l'allure des courbes basées sur différentes sources de données rend assez aisé l'énoncé d'une conclusion relative au sens, sinon au niveau, de l'erreur de complêtude du recensement de 1986, par âge et sexe. Il semble clair que, pour tous les groupes d'âge et pour les deux sexes, il y a sousdénombrement net. Le niveau du sous-dénombrement varie selon l'âge mais atteint un point culminant pour les 20-24 ans et demeure important pour les 25-29 ans, surtout pour les hommes (il est moindre pour les femmes). Cependant, la correction du recensement eu égard aux erreurs de complétude nécessite la détermination d'un niveau d'écart fixé pour chaque groupe d'âge et chaque sexe. L'utilisation des erreurs obtenues par comparaison avec l'une ou l'autre source de données ne devrait pas porter à consêquence pour la majorité des groupes d'âge. Ce n'est cependant pas le cas pour tous, en particulier pour les 15-19 et les 20-24 ans, ainsi que pour les 65 ans et plus.

Il nous faut donc, avant de trancher, chercher le pourquoi des écarts entre les courbes et trouver les facteurs susceptibles d'expliquer nos observations précédentes. 


\section{FACTEURS EXPLICATIFS DES ECARTS ENTRE ERREURS DE COMPLETUUDE}

Nous avons ajusté au meilleur de notre connaissance les différentes sources de données, et le recensement lorsque c'était nêcessaire, tant pour la mesure de l'erreur de complétude que pour les populations ciblées. Néanmoins, il demeure que certaines corrections n'ont pu être faites qu'en partie (par exemple celles que nécessite le passage du sous-dénombrement brut selon la CVD à l'erreur de complêtude, et celles que suppose l'ajustement des populations cibles selon le recensement et la sécurité de la vieillesse); d'autre part, la raison d'être même des sources et leur conception diffèrent. Il en résulte que plusieurs facteurs peuvent expliquer les écarts observés entre elles. Ce sont la méthode utilisée, les corrections effectuées, la dépendance des sources comparées et les erreurs contenues dans les sources tierces.

\section{Méthode utilisee}

Pour évaluer la qualité du recensement de 1986, par âge et sexe, nous avons fait l'hypothèse que la source de donnees à laquelle ce recensement est comparé représente la population cible recherchée, soit la population canadienne "réelle" au ler juin 1986. Si cette hypothèse étalt vérifiée dans tous les cas, les courbes par groupe d'âge des erreurs de complétude seraient juxtaposées, ce qui n'est évidemment pas le cas. Les erreurs relevées sont donc non seulement attribuables au recensement mais également à certaines (advenant qu'une des sources représente la population "réelle») sinon à toutes les sources tierces. Comment faire la part de ce qui est imputable à l'un et aux autres ? Les observations suivantes pourraient nous aider à y parvenir.

\section{Corrections effectuées}

Dans la première partie de cet article, nous avons ajustẻ les données provenant des différentes sources utilisées comme du recensement, et ce pour deux fins : l'obtention de l'erreur de complétude et la comparabilité des populations cibles. La plupart de ces ajustements ont été faits sur la base d'hypothèses plus ou moins solides ou vérifiables. Une marge d'erreur entoure donc chacun des chiffres utilisés dans nos calculs des erreurs de complétude. En bref, ces ajustements sont : 
- la réduction de $5,4 \%$ du sous-dénombrement brut mesuré par la contre-vérification des dossiers de 1981 (estimations postcensitaires définitives) et de 1986, afin d'obtenir l'erreur de complétude;

- la majoration des naissances qui sont à la base de l'estimation par composantes (générations postérieures à 1961) pour prendre en compte le sous-enregistrement, parfois élevé;

- l'estimation des récipiendaires des allocations familiales en Ontario;

- la déduction des chiffres d'AF des récipiendaires étrangers présents au Canada mais non ciblés par le recensement;

- la répartition par sexe des personnes qui sont récipiendaires de la sécurité de la vieillesse via le Régime de pensions du Canada;

- l'ajustement des effectifs recensés, visant à ne conserver que les personnes admissibles à la sêcurité de la vieillesse.

Les deuxième, troisième et quatrième corrections nous semblent solides et basées sur des chiffres et méthodes éprouvés. Les trois autres ne le sont pas. Le taux estimé de surdênombrement brut est fragile, car il est basé sur une seule étude à faible échantillon. La répartition par sexe des récipiendaires du Programme de sécurité de la vieillesse via le Régime de pensions du Canada, faite au prorata des autres récipiendaires domestiques du PSV, peut être inexacte, puisque seules les personnes ne recevant pas de supplêment de revenu garanti restent dans ce groupe et que les femmes ont généralement des revenus moindres que les hommes. Il en résulterait une sous-estimation des hommes retraités de 80 ans et plus et une surestimation des femmes du même groupe d'âge. Enfin, comme on l'a vu plus haut, nous n'avons pu enlever du recensement l'ensemble des personnes non ciblées par la sécurité de la vieillesse, ce qui pourrait faire surestimer quelque peu les effectifs recensés comparés à cette source précise.

\section{Dépendance entre le recensement et une source tierce}

Une des sources utilisées n'est pas indépendante du recensement, ce qui peut fausser l'analyse qui en est faite. En effet, les estimations postcensitaires définitives de la population canadienne reposent sur les effectifs recensés en 1981. Elles conservent donc les erreurs inhérentes à un recensement, telles les erreurs de réponse, d'imputation, de traitement ou de classification. 


\section{Erreurs dans les sources tierces}

Malgré leur qualité certaine, les sources tierces utilisées pour dêterminer les populations cibles sont également imparfaites à divers degrés.

Dans une étude récente, R. D. Burgess (1988) décrit les sources potentielles d'erreurs de la contre-vêrification des dossiers, les plus importantes étant les erreurs d'échantillonnage et le biais de non-réponse découlant du nondépistage de certaines personnes recherchées. L'erreur d'échantillonnage de 1986, mesurée par le coefficient de variation (l'erreur type, divisée par le taux estimé de sousdénombrement brut), est présentée, par groupe d'âge et par sexe, au tableau 11. L'indice fluctue de 3,6\% (total, sexes réunis) à $30,6 \%$ (hommes, 65 ans et plus). Plus le groupe étudié est petit, plus l'erreur d'échantillonnage est grande. Étant donné la taille de cette erreur pour certains groupes d'âge, il devient difficile de commenter les différences et d'en chercher le pourquoi.

Ces erreurs d'échantillonnage et ce biais de non-réponse, de même que les erreurs inhérentes à un recensement, sont êgalement présents dans les estimations postcensitaires de la population, celles-ci reposant sur le recensement antérieur corrigé. S'y ajoutent, en particulier pour les estimations par composantes (population née depuis 1961), les erreurs sur les composantes, notamment la migration. En effet, si les données

TABLEAU 11

Erreur d'échantillonnage (\%) de la contre-vérification des dossiers ${ }^{\mathrm{a}}$ du recensement de 1986, par groupe d'âge et sexe, Canada

\begin{tabular}{lrrr}
\hline & Hommes & Femmes & Total \\
\hline $0-4$ ans & 30,2 & 25,5 & 21,1 \\
$5-14$ & 15,7 & 14,9 & 12,3 \\
$15-19$ & 17,9 & 23,2 & 15,4 \\
$20-24$ & 5,5 & 9,7 & 5,0 \\
$25-34$ & 7,1 & 11,6 & 6,7 \\
$35-44$ & 15,0 & 23,4 & 13,3 \\
$45-54$ & 26,0 & 24,2 & 15,8 \\
$55-64$ & 25,0 & 22,4 & 14,8 \\
$\geq 65$ & 30,6 & 20,9 & 17,0 \\
Total & 4,1 & 5,6 & 3,6 \\
\hline
\end{tabular}

Source : Statistique Canada, 1988a : 14.

a Á l'exclusion du Yukon et des Territoires du Nord-Ouest. La population des réserves indiennes incomplètement énumérées est considérée comme "non énumérée aux fins de ce tableau. 
d'état civil relatives aux naissances et aux décès sont de bonne qualité (Statistique Canada, 1987), les données de migration internationale doivent être estimées, le Canada ne disposant d'aucun enregistrement de l'émigration. Malgré les techniques de plus en plus complexes utilisées, qui mettent en oeuvre de nombreux fichiers administratifs (allocations familiales, immigrants reçus selon Emploi et Immigration Canada), les estimations de l'êmigration sont parfois éloignées des sorties réelles (Statistique Canada, $1987: 77$ ). Ces estimations des deux types (postcensitaires et "par composantes") souffrent également des différences entre les populations ciblées par les diverses sources de données utilisées pour leur calcul (voir, plus haut, la section sur les estimations démographiques par composantes).

Comme nous l'avons souligné précédemment, de nombreuses études (Haché, 1984; Michalowski, 1988) ont montré l'excellente qualité et l'exhaustivité du fichier des allocations familiales de Santé et Bien-être social Canada. En fait, l'erreur possible proviendrait du non-enregistrement d'un enfant au fichier mais, cet enregistrement étant la condition essentielle à l'attribution de fonds, les lacunes sont faibles sinon inexistantes. Le seul problème proviendrait alors du dêlai entre la demande d'inscription et l'inscription comme telle : une naissance, une immigration ne figurent pas au fichier dès qu'elles surviennent. À l'inverse, des personnes qui auraient dû être retranchées peuvent recevoir leur redevance. Cependant, les données d'une année étant réactualisées aux six mois durant deux ans, les lacunes de ce type devraient être négligeables dans le fichier final, utilisê ici.

Le fichier de la sécurité de la vieillesse est beaucoup moins connu et sa qualité n'a pas encore vraiment été évaluée. Cependant, tout comme le fichier des allocations familiales, il souffre de délais d'enregistrement et ce, d'autant plus qu'il n'est pas rêactualisé. De plus, le taux de sous-dénombrement mesuré peut être affecté par la faible taille des effectifs dans les groupes d'âge élevés et par les mouvements migratoires importants et parfois saisonniers des retraitês.

Enfin, il importe de signaler que si le recensement et les estimations postcensitaires ou par composantes (population née depuis 1961) décrivent l'état de la population canadienne au ler juin 1986, la contre-vérification des dossiers commence dès le lendemain du jour du recensement; mais le dépistage (communication avec la personne choisie ou quelqu'un la 
connaissant, recherche des nouvelles adresses, détermination du statut de l'individu sélectionné, etc.) peut s'étaler sur des jours sinon des semaines. La personne recherchée peut alors décéder ou migrer et donc demeurer introuvable aux fins de la CVD. Quant aux données des allocations familiales et de la sécurité de la vieillesse, elles concernent les récipiendaires admissibles un mois donné.

Les quatre facteurs explicatifs que nous venons de décrire nous permettent de faire la part, dans les diverses observations présentées à la section précédente, des tendances normales de l'erreur de complétude et de celles qui sont induites par les mêthodes ou les sources.

1. Puisque toutes les approches concordent pour montrer la similitude des tendances par âge des erreurs de complétude, tant pour le sexe masculin là l'exception des 75-79 ans et des 80 ans et plus) que pour le sexe féminin et pour les sexes réunis, il semble bien qu'il s'agisse d'un postulat pour la suite de notre étude.

2. L'allure différente des valeurs obtenues à partir des fichiers de la sécurité de la vieillesse pour les homrnes de 7579 ans et de 80 ans et plus résulte vraisemblablement d'une mauvaise répartition par sexe des récipiendaires de la sécurité de la vieillesse via le Régime de pensions du Canada. Si, en vertu de l'hypothèse avancée à la section intitulée "corrections effectuées", il y a sous-estimation des hommes retraités de 80 ans et plus pour la sécurité de la vieillesse, il est normal que l'erreur de complétude augmente entre les 75-79 ans et les 80 ans et plus. Cela expliquerait également la pente beaucoup plus prononcée pour la même source de données que pour les estimations postcensitaires chez les femmes des mêmes groupes d'âge.

L'allure "réelle" de la courbe des erreurs de complétude serait donc, à ces âges, celle des estimations postcensitaires définitives.

3. Quant au niveau très particulier des erreurs obtenues à partir des données relatives à la sêcurité de la vieillesse, ils semblent découler des problèmes d'ajustement des effectifs recensés pour les seules personnes admissibles à la sécurité de la vieillesse. Puisque le recensement comparable est surestimé, l'écart entre les sources l'est aussi. Pour les 65-69 ans se greffe également le problème du sous-enregistrement des nouveaux récipiendaires dans le fichier non mis à jour de la sécurité de la vieillesse. Cependant, même conjugués, ces facteurs ne 
devraient pas génêrer un écart tel que celui qui est enregistré. Il est possible que l'émigration soit sous-estimée, ce qui entraîne une surestimation de la population postcensitaire : le niveau négatif d'erreur de complétude obtenu à partir des estimations postcensitaires définitives est donc trop êlevé.

Le niveau "réel" des 65 ans et plus serait vraisemblablement intermédiaire entre les niveaux provenant de la sécurité de la vieillesse et des estimations postcensitaires.

4. Les écarts de niveau des erreurs calculées à partir des diverses sources pour les 15-19 ans et les 20-24 ans sont plus difficiles à expliquer. Il est possible qu'une surestimation des naissances par l'état civil (soit une différence entre la population ciblée à l'état civil et la population ciblée au recensement), jointe à une sous-estimation cumulée de l'émigration, ait généré des estimations par composantes de la population née depuis 1961 trop élevées, et donc une erreur de complétude trop faible.

À l'inverse, les courbes de la contre-vérification des dossiers et des estimations postcensitaires dépendantes du recensement pourraient majorer l'erreur, comme d'ailleurs une sous-estimation du niveau du surdénombrement brut. Il semble donc probable que l'erreur "réelle " tombe quelque part entre celles dont nous disposons.

Ces quatre constatations, qui concernent la totalité des comparaisons effectuées, les deux sexes et l'ensemble des groupes d'âge, vont servir de base à la détermination des erreurs de complétude "réelles" du recensement de 1986.

\section{ESTIMATION DE LA POPULATION CANADIENNE "RÉELLE" PAR ÂGE ET SEXE AU IER JUIN 1986}

L'estimation de la population canadienne "réelle" par âge et sexe au ler juin 1986 passe nécessairement par la détermination des niveaux réels des erreurs de complétude, par âge et sexe, du recensement de 1986. Cependant, avant de décider de ces niveaux, nous tenons à spécifier que leur choix, comme le calcul de la population canadienne "rẻelle" à la date du recensement, n'est qu'un exercice visant à montrer la possibilité de rectifier les effectifs recensés en corrigeant les erreurs de complétude. L'imprécision qui entoure à l'heure actuelle les erreurs calculées précédemment, peu importe les sources utilisées, rend aléatoire l'utilisation des résultats qui suivent. 


\section{Détermination des niveaux réels des erreurs de complétude}

Pour faire suite aux commentaires de la section précédente, les niveaux réels des erreurs de complétude du recensement de 1986 sont déterminés comme suit, par âge et sexe :

1. Pour les 0-4, 5-9 et 10-14 ans, les niveaux observés sont similaires pour les différentes approches utilisées. Le niveau "réel" sera donc, pour chaque groupe d'âge, la moyenne des niveaux des différentes approches.

2. Pour les 15-19 ans et les 20-24 ans, les écarts sont plus prononcés. Mais comme l'erreur "réelle " semble intermédiaire par rapport aux erreurs observées, nous ferons également la moyenne de ces dernières.

3. Pour les $25-29$ ans à $60-64$ ans inclusivement, les courbes de la contre-vérification des dossiers et des estimations postcensitaires définitives se chevauchent. Puisque aucune des courbes n'est plus régulière que l'autre, nous en ferons également la moyenne.

4. Pour les 65 ans et plus, nous maintiendrons la tendance à la baisse observée pour les estimations postcensitaires définitives, en en conservant la pente mais en diminuant les niveaux d'un point de pourcentage partout, de façon à diminuer l'écart par rapport à la courbe de la sécurité de la vieillesse.

Les niveaux réels des erreurs de complêtude par âge et sexe ainsi déterminês figurent au tableau 12 . On remarque que le recensement souffre d'un sous-dénombrement net pour tous les groupes d'âge (sauf pour les femmes de 65 à 69 ans) et que la tendance par âge est similaire pour les hommes et les femmes, mais moins accentuée pour ces dernières. La taille de l'erreur de complêtude diminue des 0-4 ans aux 10-14 ans (passant de $2,51 \%$ à $1,17 \%$ chez les hommes, et de $2,35 \%$ à $1,43 \%$ chez les femmes), puis augmente rapidement et culmine chez les 20 24 ans, à $7,76 \%$ pour les hommes et à $4.95 \%$ pour les femmes. Les niveaux ne sont pas très éloignés pour les $25-29$ ans $(7,02 \%$ et $4,64 \%$ respectivement). Par la suite, la courbe, de forme logarithmique, revient à son niveau premier avant de remonter, après 65 ans, atteignant $1,66 \%$ pour les hommes et $1,43 \%$ pour les femmes à 80 ans et plus.

\section{Population canadienne réelle par âge et sexe au ler juin 1986}

Les erreurs de complêtude "réelles* du recensement de 1986 êtant connues, il est possible de corriger les effectifs recensés. 
TABLEAU 12

Estimation de l'erreur de complétude réelle du recensement de 1986, selon l'âge et le sexe, Canada

\begin{tabular}{lcc}
\hline & Hommes & Femmes \\
\hline $0-4$ ans & $-2,51$ & $-2,35$ \\
$5-9$ & $-1,72$ & $-1,44$ \\
$10-14$ & $-1,17$ & $-1,43$ \\
$15-19$ & $-2,23$ & $-1,93$ \\
$20-24$ & $-7,76$ & $-4,95$ \\
$25-29$ & $-7,02$ & $-4,64$ \\
$30-34$ & $-3,71$ & $-2,71$ \\
$35-39$ & $-3,09$ & $-1,56$ \\
$40-44$ & $-3,61$ & $-1,43$ \\
$45-49$ & $-2,93$ & $-1,29$ \\
$50-54$ & $-1,70$ & $-1,38$ \\
$55-59$ & $-2,13$ & $-1,89$ \\
$60-64$ & $-1,30$ & $-1,75$ \\
$65-69$ & $-0,38$ & $+0,65$ \\
$70-74$ & $-0,80$ & $-0,53$ \\
$75-79$ & $-1,41$ & $-1,29$ \\
$\geq 80$ & $-1,66$ & $-1,43$ \\
\hline
\end{tabular}

Source : tableau 10. Calculs effectués à la Division de la démographie de Statistique Canada.

En effet, puisque

l'erreur de complétude $=\frac{\text { population recensée }- \text { population ciblée }}{\text { population ciblée }}$ alors,

la population ciblée $=\frac{\text { population recensée }}{1+\text { erreur de complétude }}$

la population ciblée étant la population "réelle" recherchée.

Les effectifs recensés corrigés figurent au tableau 13, par groupe d'âge et par sexe. La population canadienne totale, au ler juin 1986, aurait été de 26062580 personnes, distribuées à raison de $49,6 \%$ d'hommes et $50,4 \%$ de femmes. Le groupe d'âge le plus nombreux, tant chez les hommes que chez les femmes, est celui des 25-29 ans, mais il est peu différent des 2024 ans. L'âge moyen de la population est de 33,3 ans pour les hommes et de 35,3 ans pour les femmes.

L'effet de la correction, outre l'ajout de 709624 personnes (422 357 hommes et 287267 femmes), modifie légèrement la structure par sexe, la proportion d'hommes passant de $49,3 \%$ à 
TABLEAU 13

Estimation de la population canadienne réelle et distribution pour 10 000, par âge et sexe, Canada, 1er juin 1986

\begin{tabular}{|c|c|c|c|c|c|c|}
\hline & \multicolumn{3}{|c|}{ Population canadienne réelle } & \multicolumn{3}{|c|}{ Distribution pour 10000} \\
\hline & $\mathrm{H}$ & F & Total & $\mathrm{H}$ & $\mathbf{F}$ & Toltal \\
\hline $0-4$ ans & 954611 & 906557 & 1861168 & 366 & 348 & 714 \\
\hline $5-9$ & 938985 & 890284 & 1829269 & 360 & 341 & 701 \\
\hline $10-14$ & 930436 & 885324 & 1815760 & 357 & 340 & 697 \\
\hline 15-19 & 1010626 & 960879 & 1971505 & 388 & 369 & 757 \\
\hline $20-24$ & 1229046 & 1182653 & 2411699 & 472 & 454 & 926 \\
\hline $25-29$ & 1254910 & 1235588 & 2490498 & 481 & 474 & 955 \\
\hline $30-34$ & 1127025 & 1133926 & 2260951 & 432 & 435 & 867 \\
\hline $35-39$ & 1044407 & 1032142 & 2076549 & 401 & 396 & 797 \\
\hline $40-44$ & 842195 & 816231 & 1658426 & 323 & 313 & 636 \\
\hline $45-49$ & 680745 & 665228 & 1345973 & 261 & 255 & 516 \\
\hline $50-54$ & 627639 & 622412 & 1250051 & 241 & 239 & 480 \\
\hline $55-59$ & 607148 & 621941 & 1229089 & 233 & 239 & 4.72 \\
\hline $60-64$ & 537903 & 605708 & 1143611 & 206 & 232 & 438 \\
\hline $65-69$ & 416545 & 494391 & 910936 & 160 & 190 & 350 \\
\hline $70-74$ & 327269 & 416479 & 743748 & 126 & 160 & 286 \\
\hline $75-79$ & 213110 & 304544 & 517654 & 82 & 117 & 199 \\
\hline$\geq 80$ & 187841 & 357852 & 545693 & 72 & 137 & 209 \\
\hline Total & 12930441 & 13132139 & 26062580 & 4961 & 5039 & 10000 \\
\hline \multicolumn{4}{|c|}{ Àge moyen } & 33,31 & 35,27 & \\
\hline
\end{tabular}

Sources : tableau 1 et tableau 12. Calculs effectués à la Division de la dëmographie de Statistique Canada. Données inédites.

49,6\%. L'âge moyen, quant à lui, diminue pour les deux sexes, passant de 33,4 ans à 33,3 ans pour les hornmes et de 35,4 à 35,3 ans pour les femmes. Les groupes d'âge étant touchés différemment par les erreurs de complétude, la structure par âge se trouve évidemment modifiée par les corrections, comme le montrent les tableaux 1 et 13. En gros, les jeunes (0-14 ans) et les plus de 50 ans perdent de leur importance dans l'ensemble de la population canadienne. Il s'ensuit que le rapport de dépendance ${ }^{11}$ diminue, passant de 0,455 à 0,443 pour les hommes, de 0,486 à 0,479 pour les femmes, et de 0,470 à 0,461 pour les sexes réunis.

\section{CONCLUSION}

Le recensement est certainement la source de données la plus utilisée lorsqu'on veut connaître la population canadienne. Il n'est cependant pas exempt d'erreurs et son bon

$11 \quad \frac{\text { Effectifs 0-14 et } 65 \text { ans et plus }}{\text { Effectifs } 15-64 \text { ans }}$ 
emploi passe par une juste connaissance du niveau et du sens de ces erreurs. À cette fin, Statistique Canada effectue la contrevêrification des dossiers à brève échéance après la date du recensement, et compile sur cette base des taux de sousdénombrement brut selon diverses caractéristiques des individus, dont l'âge et le sexe. Pour être réalistes, ces taux doivent cependant être corrigés en fonction $\mathrm{du}$ surdẻnombrement brut, malheureusement mal connu. Le recensement de 1986 est en effet le premier pour lequel le surdẻnombrement a été étudié, avec un faible échantillon cependant. Sachant que l'expérience sera reconduite en 1991 avec un êchantillon plus important, nous espérons que les niveaux obtenus seront alors plus fiables.

Néanmoins, corrigés en fonction du niveau de surdénombrement brut mesuré pour 1986, les taux de sousdénombrement induits par la contre-vérification des dossiers semblent aller de pair avec ceux d'autres sources: les estimations postcensitaires dêfinitives; les estimations par composantes (générations nées depuis 1961); et les données sur les récipiendaires d'allocations familiales et de la sécurité de la vieillesse. L'évolution par âge des niveaux de complétude est identique d'une source de données à l'autre, même si la valeur mème des niveaux diffère parfois. Chaque source utilisée étant passible d'erreurs de diverses sortes et aucune ne s'affirmant plus exacte que les autres, dans cet exercice nous avons êtabli comme erreur de complétude la moyenne des taux obtenus à partir des diverses sources (sauf pour les 65 ans et plus). Sur cette base, nous avons estimé l'effectif "réel" de la population canadienne, par âge et sexe, au ler juin 1986.

Il est évident que ces chiffres, basés sur des hypothèses et décisions non toujours prouvées ou étayées, sont entachés d'erreurs. Le but de cet exercice était cependant de montrer qu'il est possible de corriger les données du recensement et de se rapprocher ainsi de la population "réelle" qu'il nous faut utiliser dans nos études et analyses. Malheureusement, à l'heure actuelle, il ne peut s'agir que d'un exercice.

En effet, une correction exacte nécessite des progrès dans la connaissance des erreurs de complétude du recensement. La comparaison des effectifs recensés à ceux d'autres sources semble être d'une utilitê considérable. Elle ne sera cependant parfaite que si l'on parvient à obtenir dans tous les cas des effectifs comparables pour les deux sexes et les mêmes groupes d'âge. Il serait, de plus, important que soient développés de 
nouveaux fichiers administratifs canadiens aux fins d'usage statistique : la réactualisation pour un mois donné du fichier de la sécuritê de la vieillesse serait un bon exemple de mesure utile.

Le recensement est un outil de base pour toute étude sociale. Il importe cependant qu'il soit exact ou que, du moins, ses lacunes soient bien connues et mesurées, afin que l'utilisateur puisse dêcider, en fonction de l'usage qu'il en fait, s'il doit le corriger ou non. Les erreurs de complétude peuvent créer des distorsions importantes dans la structure de la population et gênérer des conclusions fausses sur les modifications des tendances dans le temps. Améliorer la mesure des erreurs de complétude, c'est améliorer la qualité des données et, par ricochet, la qualité de la connaissance de l'état et de l'évolution de la population canadienne. C'est pourquoi, alors qu'actuellement le recensement du Canada n'est pas corrigé en fonction des erreurs de complétude, il serait souhaitable que du moins les estimations postcensitaires de la population. le soient. Cela pourrait être possible, au niveau du Canada en tout cas, mais les erreurs de complétude devront être connues de façon plus sûre et plus détaillée.

\section{REFERENCES BIBLIOGRAPHIGUES}

ACDI, 1988. Rapport annuel 1986-1987. Ottawa.

BURGESS, R.D., 1988. "Évaluation des estimations du sousdénombrement obtenues par la contre-vérification des dossiers du recensement du Canada». In Techniques d'enquête, 14, 2 (décembre), 147-167.

CHOI, C.Y., D.G. STEEL et I.J. SKINNER, 1988. ^Redressement des chiffres du recensement de 1986 en Australie pour le sousdénombrement.. In Techniques d'enquête, 14, 2 (décembre), 187204.

EMPLOI ET IMMIGRATION CANADA, 1980. Rapport annuel 1978. Ottawa.

FAY, R.E., J.S. PASSEL, G.J. ROBINSON et C.D. CONRAN, 1988. The Coverage of Population in the 1980 Census. United States Department of Commerce, Bureau of the Census.

HACHE, J.-G., 1984. Vers un système universel de comptabilité de la migration internationale: l'exemple du Canada. Université de Montreal, thèse de maîtrise.

LAPIERRE, E., 1971. Estimation, à l'aide des techniques d'analyse démographique, du sous-dénombrement net au recensement suivant l'áge et le sexe. Statistique Canada, Section de l'analyse démographique et de la recherche, document de travail. 
Lot sur les allocations familiales, Canada, 1973, révision du 15 août 1980.

Loi sur la sécurité de la vieillesse, ch. 0-9, Canada, 1985.

Lois révisées du Canada de 1985.

MICHALOWSKI, M., 1988. An Estimation of Returning Canadian Residents After Staying Abroad for One Year or More. A Feasibility Study. Ottawa, Statistique Canada, Division de la démographie.

REDFERN, P., 1981. «Census 1981. An Historical and International Perspectiven. In Population Trends (printemps), 3-15.

ROMANIUC, A., 1988. ، Une approche démographique à l'évaluation du recensement de 1986 et des estimations de population pour le Canada ". In Techniques d'enquête, 14, 2 (décembre), 169-185.

STATISTIQUE CANADA, 1987. Méthodes d'estimation de la population, Canada. No 91-528F au catalogue, Ottawa, $117 \mathrm{p}$.

STATISTIQUE CANADA, 1988a. "Taux de sous-dénombrement provenant de la contre-vérification des dossiers de 1986". In Bulletin d'information à l'intention des utilisateurs, 2 (juillet), 24 p.

STATISTIQUE CANADA, 1988b. Manuel de référence du recensement de 1986. No 99-104F au catalogue, Ottawa, $150 \mathrm{p}$.

STATISTIQUE CANADA, 1990. Qualité des données: couverture. No $99-135 \mathrm{~F}$ au catalogue, Ottawa, $92 \mathrm{p}$. 


\section{RÉSUMÉ - SUMMARY — RESUMEN}

\section{FORTIER Céline et Ronald RABY - ÉVALUATION DE LA QUALTÉ DES DONNÉES PAR ÂGE ET SEXE DU RECENSEMENT DE 1986 AU NIVEAU NATIONAL}

Le recensement est certainement la source d'information la plus complète sur la population canadienne. Il n'est cependant pas exempt d'erreurs, en particulier de l'erreur de complétude. Statistique Canada évalue le niveau de cette dernière erreur, au total et pour certaines caractéristiques, au moyen de la contre-vérification des dossiers. Malheureusement, cette méthode ne permet la mesure que du sousdénombrement brut. La présente étude a pour but de comparer l'estimation des erreurs de complétude par âge et sexe ainsi obtenues à l'échelle nationale en 1986 à celles qui résultent de la comparaison du recensement à d'autres sources de données : estimations postcensitaires définitives; estimations par composantes (générations nées depuis 1961); et fichiers des réciplendaires d'allocations familiales ( $0-14$ ans) ou de sécurtté de la viellesse (65 ans et plus). L'évolution par âge des niveaux de complétude ainsi calculés est identique d'une source de données à l'autre, même si la valeur des niveaux diffère parfois. La moyenne des valeurs pour chaque âge et chaque sexe a permis, à titre d'exercice, d'estimer l'effectif séel. de la population canadienne au ler juin 1986.

\section{FORTIER Céline et Ronald RABY - EVALUATION OF THE QUALITY OF 1986 CENSUS DATA BY AGE AND SEX AT THE NATIONAL LEVEL}

The census is, without doubt, the most complete source of information on the Canadian population. Nevertheless, it is not free of error, especially in terms of coverage. Statistics Canada estimates this level of undercount, overall and by certain characteristics, using the Reverse Record Check (RRC). Unfortunately, this method yields only crude measures of undercoverage. The purpose of the present study is to compare the RRC estlmates of undercoverage, by age and sex, with estimates produced using other sources of data, specifically: final postcensal estimates; estimates produced by the component method (for cohorts since 1961); and estimates produced through use of administrative data from Family Allowance (ages 0-14) and Old Age Security (ages 65+). Results indicate that, even though the estimates of the level of undercoverage by age differ depending on the source and/or method, the same general trend is observed. Using the average of such figures, for example, can yield an estimate of the "actual" size of the Canadian population as of June 1, 1986. 
FORTIER Céline y Ronald RABY - EVALUACIÓN DE LA CALIDAD DE LOS DATOS POR EDAD Y SEXO DEL CENSO DE 1986 A NIVEL NACIONAL.

El censo es seguramente la fuente de información la más completa sobre la plobactón canadiense. No está sin embargo exento de errores, y particularmente del error de complettud. Estadistica Canadá evalúa el nivel de este último error, en su totalidad y para algunas caracteristicas, por medio de la contra-verificación de los expendientes. Por desgracia, este método permite únicamente medir el subrecuento bruto. El presente estudio tiene como objetivo de comparar la estimación de los errores de complettud por edad y sexo obtenidos de esta manera a escala nacional en 1986 con aquellos que resultan de la comparación del censo de otras fuentes de datos: estimaciones post-censales definitivas; estimaciones por componentes (generaciones nacidas desde 1961); y ficheros de los rectpiendarlos de alocaciones familiares (0-14 anos) o de segurtiad de la vejez (65 anos y más). La evolución por edad de los niveles de completitud asi calculados es idéntica de una fuente de datos a otra, aún st a veces el valor de los niveles difiere. El promedio de los valores por cada edad y cada sexo ha permitido, a título de ejercicio, de estimar el efectivo real de la población canadiense el $1^{\text {ro }}$ de junio de 1986. 